\title{
Geometric interpretation of the Zero-Moment Point
}

\author{
Gijs van Oort* and Stefano Stramigioli*
}

\begin{abstract}
In this article we show that the concept of screws and wrenches gives us tools to geometrically establish the relation between the ground reaction wrench and the ZeroMoment Point. In order to arrive at this, we show how a wrench can be decomposed into separate components. The proposed method gives a general, completely coordinate-free way to find the $\mathrm{ZMP}$ and contributes in improving the geometrical insight.
\end{abstract}

\section{INTRODUCTION}

The Zero-Moment Point (ZMP) [1], is widely known in the area of walking robots. Numerous researchers have investigated this point and have given their own definitions and interpretations of it (for references, see section II). The theory on screws, introduced by Ball [2] in 1900 is a $6 \mathrm{D}$ generalization of velocity and force, being the Twist and Wrench respectively. This approach is coordinate-free, meaning that the equations and their results are invariant for the choice of coordinates. The absense of coordinates gives greater flexibility (the equations work for any orientation of the robot; they are not limited to a certain pose in the world), reduces the chance of errors (e.g., one cannot be tempted to make assumptions like 'the robot will walk approximately in $\mathrm{x}$-direction, so let's use the $\mathrm{x}$-coordinate for the distance traveled' because the lack of coordinates implies that no x-direction was defined in the first place), and most importantly, mimics what happens in nature: nature does not have coordinates at all; the laws are completely dictated by the physics of the system, by nothing more. The concept is also called 'geometric dynamics', emphasizing the fact that everything follows from the geometric properties of the system (in a broad sense, e.g., geometric mass and force distribution), not from the way you look at the system (i.e., which coordinates you use).

In this paper we combine the knowledge of the ZeroMoment Point and screw theory, to give a novel, geometric interpretation of the Zero-Moment Point. We will show how the position of the ZMP can be found from the ground reaction wrench (generalization of the ground reaction force) and geometric rules on wrench decomposition. This leads to more insight in the position of the ZMP and how this relates to the ground wrench exerted on the foot.

The relation between ground reaction wrench and ZMP has been (indirectly) presented before [3], [4], [5], but without any discussion nor proof. The mathematical expression for the ZMP has been known for over 40 years now, so

\footnotetext{
This work has been funded by the European Commission's Seventh Framework Programme as part of the project VIACTORS under grant no. 231554.

* $\{$ g.vanoort,s.stramigioli $\} @$ utwente.nl, Department of Electrical Engineering, Faculty of Electrical Engineering, Mathematics and Computer Science, University of Twente, 7500 AE Enschede, The Netherlands.
}

in that sense the final result of this paper is not new. The insightful, graphical way of achieving the result however is the real contribution of this paper.

In sections II and III we introduce the key terms of this paper further. In section IV we address wrench decomposition. In section $\mathrm{V}$ we present the main contribution of this article, being the geometric relation between the ground reaction wrench and the ZMP. In section VI it will be shown that this leads to a simple explicit expression for the ZMP position. Conclusions are discussed in section VII.

\section{The Zero-Moment Point}

The Zero-Moment Point, ZMP, was introduced by $\mathrm{M}$. Vukobratović and D. Juričić around 1969 [6], [7] (although the term ZMP was only introduced a few years later). It should be noted that "...the notion of ZMP has never been introduced in the form of a formal definition..." [1]. This has given rise to dozens of researchers giving their own definitions and interpretations to this point. In general, the definitions of the ZMP can be divided into two groups:

1) Definitions in which the position of the ZMP is related to gravity and inertia forces, e.g.:

- "The ZMP is defined as that point, on the ground at which the net moment of the inertial forces and the gravity forces has no component, along the horizontal axes." [8]

- "The ZMP is the point on the ground where the tipping moment acting on the biped, due to gravity and inertia forces, equals zero, the tipping moment being defined as the component of the moment that is tangential to the supporting surface." [5]

2) Definitions in which the position of the ZMP is related to the ground-reaction force acting on the robot, e.g.:

- "The ZMP (Zero-Moment Point) is defined to be a point on the ground at which the tangential component of the moment generated by the ground reaction force/moment becomes zero." [9]

- "The pressure under supporting foot can be replaced by the appropriate reaction force acting at a certain point of the mechanism's foot. Since the sum of all moments of active forces with respect to this point is equal to zero, it is termed the Zero-Moment Point (ZMP)."1 ${ }^{1}[10]$

\footnotetext{
${ }^{1}$ At first glance, this definition seems incorrect because it is well-known that the existence of a moment around the ZMP $i$ s allowed as long as it is has no component along the ground surface. However, the author (the inventor of the ZMP) cleverly chose to use the 'pressure under supporting foot', which, by definition, only has a component perpendicular to the ground surface. This indeed results in an appropriate reaction force at the ZMP having no moment at all. The friction forces along the ground surface, that would give a vertical moment around the ZMP, were simply ignored.
} 

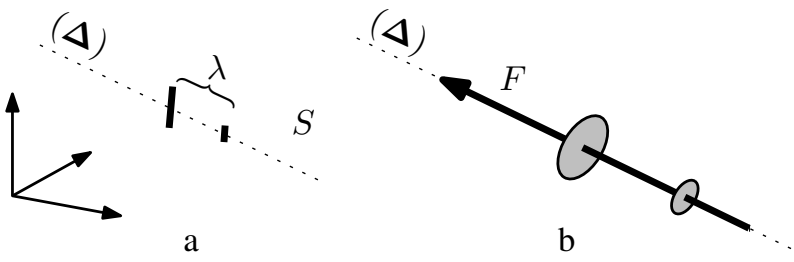

Fig. 1. a) Graphical representation of a screw $S$. The pitch $\lambda$ is shown with two small ticks on the axis (direction from the large to the small disk). b) Graphical representation of a wrench in a 3D space: the magnitude of force $F$ along axis $(\boldsymbol{\Delta})$ is indicated by the length of the arrow. The magnitude of moment $M$ along $(\boldsymbol{\Delta})$ is indicated by the distance between the two discs (direction from the large to the small disc).

Interestingly, both cited definitions in group 1 (as well as most others in this group) fail to include external disturbance forces (such as someone pushing the robot), which makes these definitions incomplete. After fixing this (for example, the first definition would become: The ZMP is defined as that point, on the ground at which the net moment of the inertial forces, the external disturbance and gravity forces has no component, along the horizontal axes), all definitions are correct and (necessarily) will give the same location for the ZMP. This location is equal to the location of the Center of Pressure (CoP). A sufficient amount of papers have already been written to prove all this [5], [11] ${ }^{2}$, so we will not repeat that.

The ZMP lying strictly within the support polygon only tells us that the stance foot (or feet, if the robot is in doublesupport phase) will not start to rotate about one of its edges. This is neither a sufficient nor a necessary condition for walking without falling [12]. It must be said however, that having the ZMP strictly within the support polygon makes continuous pose control of the robot a lot easier ${ }^{3}$, which is probably the reason that so many people think that it is the only solution.

\section{WRENCH - A 6D FORCE}

In this paper we will use the widely known concept of wrenches (a 6D generalization of a force), from geometric mechanics [2], [13].

We will first introduce the notion of a screw. A screw $S$ consists of an (undirected) axis $(\boldsymbol{\Delta})$ in space and an associated scalar $\lambda \in \mathbb{R}$ called the pitch (the unit of $\lambda$ is [m]). The axis can be fully specified by the position $r$ of some point on the axis and a (unit) direction vector $\omega$, such that $(\boldsymbol{\Delta})=\{r+\alpha \omega \mid \alpha \in \mathbb{R}\}$. A graphical representation of a screw is shown in figure 1a. Because the axis is undirected, the screw $S=(\omega, r, \lambda)$ is the same screw as $S^{*}=(-\omega, r, \lambda)$.

A wrench $W$, having intensity $v$, on a screw $S$ (having axis $(\boldsymbol{\Delta})$ and pitch $\lambda$ ) can be interpreted as a combination

\footnotetext{
${ }^{2}$ In these papers it was assumed that there are no external disturbances, but the results still hold if there are.

${ }^{3}$ When the foot is rotating about one of its edges, we essentially have an underactuated system, which is hard to control exactly. Luckily, for walking without falling, we believe it is not necessary to exactly control the robot's pose at all times.
}
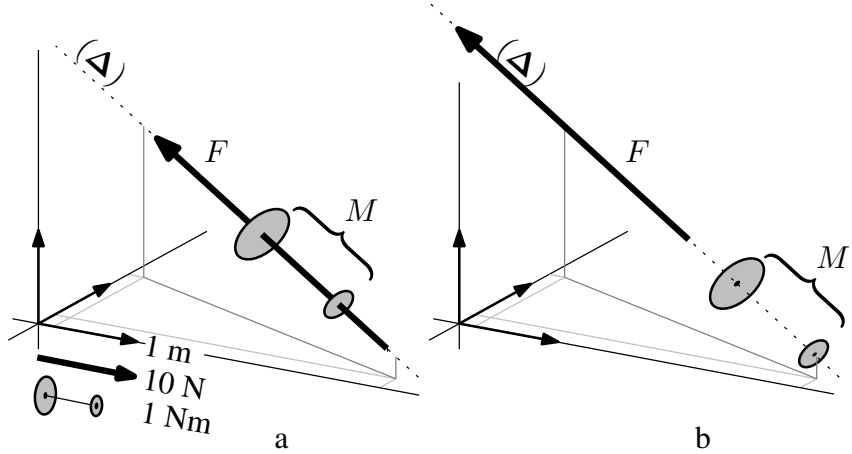

Fig. 2. a) Introduction of reference lengths. In the lower left, a scale for meter, Newton and Newton meter are given. It can be deduced that this wrench has $|F|=35 \mathrm{~N},|M|=2.4 \mathrm{Nm}$ and (thus) $\lambda=-0.07 \mathrm{~m}$. The vector and discs can (independently) be freely translated along the axis without losing meaning, hence b) represents the same wrench. We will use the scaling shown in this figure for the other figures in this paper.

of:

1) A (linear) force $F=v \cdot \omega$ along axis $(\Delta)$, and

2) A (rotational) moment $M=\lambda F=\lambda v \cdot \omega$ along axis $(\Delta)$.

The pitch $\lambda$ is the ratio between amount of moment (in [Nm]) and amount of force (in $[\mathrm{N}]$ ). In order to specify the direction of the force (either towards one end of the axis or towards the other), we will use the direction of vector $\omega$ : if the force goes into the direction of $\omega$, we will denote a positive $v$, otherwise a negative one. A graphical representation of a wrench is shown in figure $1 \mathrm{~b}$. In order to have a meaning, 'reference lengths' must be provided, as shown in figure 2.

The numerical expression of the wrench depends on the choice of coordinates, thus on the chosen reference frame $\Psi_{\text {ref }}$. Hence, when expressing a wrench in numbers, the choice of coordinates must always be given, otherwise it is unclear what the numbers actually mean. In this paper we use a pre-pended superscript to express the reference frame, in the following way:

$$
{ }^{a} W \text { : A wrench expressed in reference frame } \Psi_{a} .
$$

Note that it is necessary to denote the reference frame only when a wrench is expressed in numbers; not when drawing the wrench graphically (which makes drawing wrenches completely coordinate-free) or manipulating the equations.

One way to numerically represent a wrench is in so-called Plücker coordinates [14]:

$$
{ }^{\mathrm{ref}} W=\left(\begin{array}{c}
m \\
f
\end{array}\right)=v(\underbrace{\left(\begin{array}{c}
r \wedge \omega \\
\omega
\end{array}\right)}_{\text {linear force }}+\underbrace{\lambda\left(\begin{array}{c}
\omega \\
0
\end{array}\right)}_{\text {moment }}),
$$

where $v$ refers to the intensity of the wrench, $r$ is a vector from the reference frame's origin to some point on the screw axis, $\omega$ is the unit vector indicating the direction of the axis and $\lambda$ is the pitch. The $\wedge$ denotes the cross product. $m$ and $f$ are $(3 \times 1)$ vectors. The coordinates $\left(\begin{array}{c}m \\ f\end{array}\right)$ can be interpreted as follows: the wrench $W$ is equal to a linear force $f$ exerted at the origin of the reference frame, plus a moment $m$. 


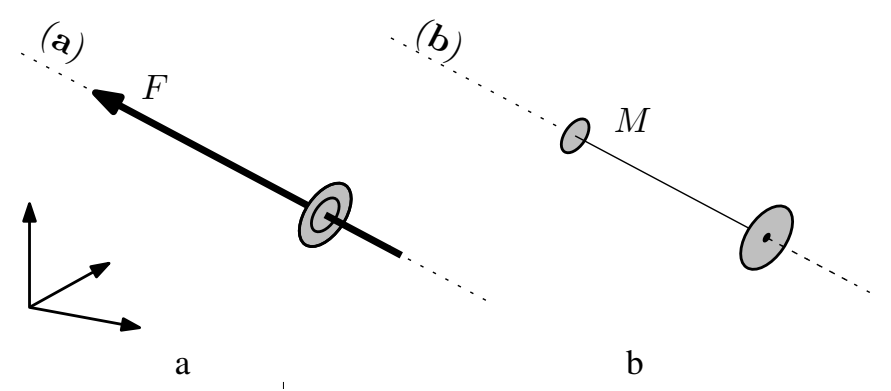

Fig. 3. a) A wrench representation of a pure force $(\lambda=0)$. b) A wrench representation of a pure moment $(\lambda=\infty)$. For reference lengths, see figure 2 .

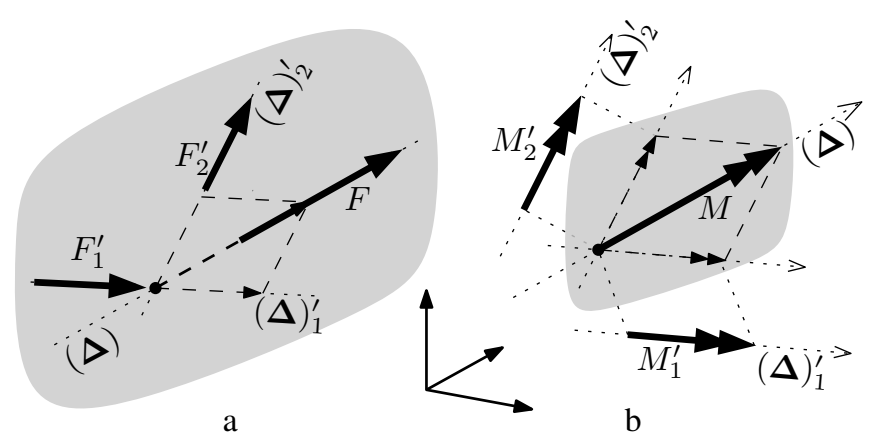

Fig. 4. a) A linear force $F$, decomposed into two components $F_{1}^{\prime}$ and $F_{2}^{\prime}$. b) A moment $M$, decomposed into two components $M_{1}^{\prime}$ and $M_{2}^{\prime}$.

A pure force $F$ along an axis (a) can be represented by a wrench with intensity $v=|F|$ along a screw with axis (a) and zero pitch. In Plücker coordinates this results in:

$$
{ }^{\text {ref }} W^{\text {force }}=v\left(\left(\begin{array}{c}
r \wedge \omega \\
\omega
\end{array}\right)+0 \cdot\left(\begin{array}{l}
\omega \\
0
\end{array}\right)\right)=v\left(\begin{array}{c}
r \wedge \omega \\
\omega
\end{array}\right) .
$$

So, even though this describes a pure force, the moment part in Plücker coordinates is non-zero due to the arm of the force with respect to the coordinates used. A pure moment $M$ about an axis (b) can be represented by a wrench with intensity $v \rightarrow 0$ along a screw with axis (b) and infinite pitch $\lambda \rightarrow \infty$; such that the magnitude of the moment becomes a finite value: $v^{*}=\lambda v=|M|$. Here we introduced the pseudo-intensity $v^{*}$ in order to circumvent problems with zero intensity. In Plücker coordinates we have:

$$
{ }^{\mathrm{ref}} W^{\mathrm{mom}}=\lim _{\substack{v \rightarrow 0 \\
\lambda v \rightarrow|M|}} v\left(\left(\begin{array}{c}
r \wedge \omega \\
\omega
\end{array}\right)+\lambda\left(\begin{array}{l}
\omega \\
0
\end{array}\right)\right)=v^{*}\left(\begin{array}{c}
\omega \\
0
\end{array}\right) .
$$

In figure 3 the graphical representation of a pure force and a pure moment as wrench are shown.

The wrench is the dual of the Twist $T$, as generalized 6D velocity; i.e., expressed in coordinates of any reference frame $\Psi_{a}$, the product $\left({ }^{a} W^{T} \cdot{ }^{a} T\right) \in \mathbb{R}$ equals the power supplied by $W$.

\section{DECOMPOSITION OF A WRENCH}

The principle behind decomposition of wrench $W$ is to find wrenches $W_{1}^{\prime} \ldots W_{n}^{\prime}$ that, together, do the same work

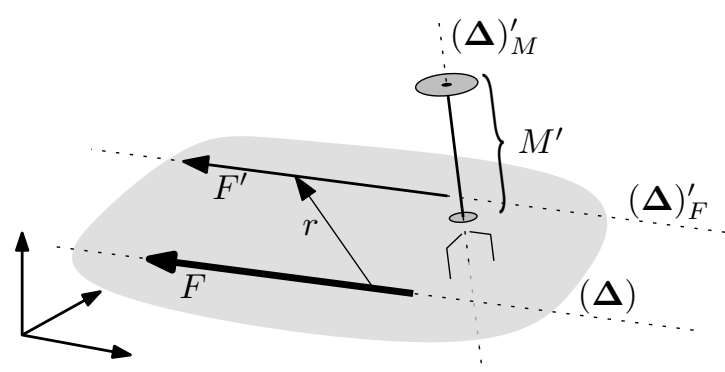

Fig. 5. Decomposition of force $F$ into (translated) force $F^{\prime}$ and moment $M^{\prime} . F$ and $r$ are not necessarily perpendicular.

as $W$ when applied on a moving rigid body for any motion. Mathematically this is not challenging at all, since wrenches represented in Plücker coordinates can be summed:

$$
{ }^{\mathrm{ref}} W=\sum_{i}\left({ }^{\mathrm{ref}} W_{i}^{\prime}\right) \text {. }
$$

Geometrically however, the problem is much more interesting: given $n$ screws $S_{1}^{\prime} \ldots S_{n}^{\prime}$, do there exist wrench intensities $v_{1}^{\prime} \ldots v_{n}^{\prime}$ such that the composition of the $n$ wrenches together yields the original wrench $W$ ?

In this section, we give conditions for the decomposition of a wrench $W$ on screw $S$ into two components $W_{1}^{\prime}, W_{2}^{\prime}$ along screws $S_{1}^{\prime}, S_{2}^{\prime}$. Decomposition into more than two forces is not considered because it is not needed in this paper. Firstly, we will review some decomposition rules for pure forces and pure moments:

DEC1 A pure force $F$ along axis $(\boldsymbol{\Delta})$ can be decomposed into pure forces $F_{1}^{\prime}$ and $F_{2}^{\prime}$ along axes $(\boldsymbol{\Delta})_{1}^{\prime}$ and $(\boldsymbol{\Delta})_{2}^{\prime}$ if and only if:

1) either the axes $(\boldsymbol{\Delta}),(\boldsymbol{\Delta})_{1}^{\prime}$ and $(\boldsymbol{\Delta})_{2}^{\prime}$ intersect each other in one point $p$ and the plane spanned by the direction vectors $\omega_{1}^{\prime}$ and $\omega_{2}^{\prime}$ (which must have distinct directions) contains the direction vector $\omega$,

2) or all axes $(\boldsymbol{\Delta}),(\boldsymbol{\Delta})_{1}^{\prime}$ and $(\boldsymbol{\Delta})_{2}^{\prime}$ are parallel and lie in the same plane. This is the limit case for $p \rightarrow \infty$ of the first condition.

If $(\boldsymbol{\Delta})_{1}^{\prime}$ and $(\boldsymbol{\Delta})_{2}^{\prime}$ are different from each other, then the decomposition is unique.

DEC2 A pure moment $M$ along an axis $(\boldsymbol{\Delta})$ can be decomposed in pure moments $M_{1}^{\prime}, M_{2}^{\prime}$ along axes $(\boldsymbol{\Delta})_{1}^{\prime},(\boldsymbol{\Delta})_{2}^{\prime}$ if and only if:

1) either the plane spanned by the direction vectors $\omega_{1}^{\prime}$ and $\omega_{2}^{\prime}$ (which must have distinct directions) contains the direction vector $\omega$,

2) or all axes $(\boldsymbol{\Delta}),(\boldsymbol{\Delta})_{1}^{\prime}$ and $(\boldsymbol{\Delta})_{2}^{\prime}$ are parallel.

In the first case, the decomposition is unique, in the second case it is not. Note that the decomposition rule for a pure force (DEC1) is a subset of the decomposition rule for a pure moment. Hence, a moment can always be decomposed along axes that fulfill the conditions for force decomposition.

DEC3 A force $F$ along axis $(\boldsymbol{\Delta})$ can be decomposed into a force $F^{\prime}$ along axis $(\boldsymbol{\Delta})_{f}^{\prime}$ and a moment $M^{\prime}$ about 
an axis $(\boldsymbol{\Delta})_{m}^{\prime}$ if and only if $(\boldsymbol{\Delta}) \|(\boldsymbol{\Delta})_{f}^{\prime}$ and $(\boldsymbol{\Delta})_{m}^{\prime}$ is perpendicular to the plane spanned by $(\boldsymbol{\Delta})$ and $(\boldsymbol{\Delta})_{f}^{\prime}$. Let $r$ be a vector from some point on $(\boldsymbol{\Delta})$ to some point on $(\boldsymbol{\Delta})_{f}^{\prime}$. Then $\left|F^{\prime}\right|=|F|$ and $M^{\prime}=-r \wedge F$. See figure 5 .

For general wrenches, things are a bit more complicated: we do not only have restrictions on the axes, but also on the pitches of each screw. Firstly, we will present two trivial cases of a wrench decomposition into two components.

Theorem 1: A wrench $W$ on screw $S$ can be decomposed into one wrench $W_{f}^{\prime}$ on screw $S_{f}^{\prime}$ representing a pure force $\left(\lambda_{f}^{\prime}=0\right)$ plus one wrench $W_{m}^{\prime}$ on screw $S_{m}^{\prime}$ representing a pure moment $\left(\lambda_{m}^{\prime}=\infty\right)$, if axis $(\boldsymbol{\Delta})_{f}^{\prime}$ coincides with $(\boldsymbol{\Delta})$ and axis $(\boldsymbol{\Delta})_{m}^{\prime}$ is parallel to $(\boldsymbol{\Delta})$.

Proof: Assume that the conditions on axes $(\boldsymbol{\Delta})_{f}^{\prime}$ (defined by $\omega_{f}^{\prime}$ and $r_{f}^{\prime}$ ) and $(\boldsymbol{\Delta})_{m}^{\prime}$ (defined similarly) are met, i.e., $\omega_{f}^{\prime}= \pm \omega ; r_{f}^{\prime}=r ; \omega_{m}^{\prime}= \pm \omega ; r_{m}^{\prime}=$ arbitrary. Without loss of generality we can assume that $\omega_{f}^{\prime}=\omega_{m}^{\prime}=\omega$ (by changing the sign of the (pseudo-)intensities $v_{f}^{\prime}$ and $v_{m}^{* \prime}$ if necessary). By expressing the wrench in Plücker coordinates, we can show that indeed there exist intensities $v_{f}^{\prime}$ and $v_{m}^{* \prime}$ that give a valid decomposition $W=W_{f}^{\prime}+W_{m}^{\prime}$ :

$$
v\left(\begin{array}{c}
r \wedge \omega \\
\omega
\end{array}\right)+\lambda v\left(\begin{array}{c}
\omega \\
0
\end{array}\right)=v_{f}^{\prime}\left(\begin{array}{c}
r_{f}^{\prime} \wedge \omega_{f}^{\prime} \\
\omega_{f}^{\prime}
\end{array}\right)+v_{m}^{* \prime}\left(\begin{array}{c}
\omega_{m}^{\prime} \\
0
\end{array}\right)
$$

so choosing $v_{f}^{\prime}=v$ and $v_{m}^{* \prime}=\lambda v$ will do. This is actually the way a wrench is built in the first place: it consists of a pure force $F=v \cdot \omega$ along axis $(\boldsymbol{\Delta})$ plus a pure moment $M=\lambda v \cdot \omega$ around this axis.

The converse of this theorem is not true (i.e., the conditions on the axes are sufficient but not necessary). As a counter-example see DEC3, where a wrench (a pure force in this case) is decomposed into a pure force and pure moment while the screws are perpendicular instead of parallel.

Theorem 2: A wrench $W$ of intensity $v$ on screw $S$, can be decomposed into two wrenches $W_{1}^{\prime}$ and $W_{2}^{\prime}$ on screws $S_{1}^{\prime}$ and $S_{2}^{\prime}$ if the axes $(\boldsymbol{\Delta})_{1}^{\prime}$ and $(\boldsymbol{\Delta})_{2}^{\prime}$ satisfy the conditions of DEC1 and the pitches satisfy $\lambda_{1}^{\prime}=\lambda_{2}^{\prime}=\lambda$.

Proof: Assume that all conditions are satisfied. Wrench $W$ consists of a force $F$ along $(\boldsymbol{\Delta})$ and a moment $M=$ $\lambda F$ around it. According to DEC1, $F$ can be decomposed into $F_{1}^{\prime}$ and $F_{2}^{\prime}$ along $(\boldsymbol{\Delta})_{1}^{\prime}$ and $(\boldsymbol{\Delta})_{2}^{\prime}$. The same holds for decomposition of $M$ into $M_{1}^{\prime}=\lambda F_{1}^{\prime}$ and $M_{2}^{\prime}=\lambda F_{2}^{\prime}$. Now $F_{1}^{\prime}$ and $M_{1}^{\prime}$ can be taken together again to form wrench $W_{1}^{\prime}$; similarly for $W_{2}^{\prime}$. A more mathematical proof is given in the appendix.

The converse of this theorem is not true: there are many sets of screws $\left\{S_{1}^{\prime}, S_{2}^{\prime}\right\}$ along which a wrench can be decomposed that do not satisfy the above conditions.

A more general theorem can be stated, which will be useful for the determination of the ZMP later on:

Theorem 3: A wrench $W$ of intensity $v$ on screw $S$, can be decomposed into a wrench $W_{f}^{\prime}$ on screw $S_{f}^{\prime}$ representing a pure force $\left(\lambda_{f}^{\prime}=0\right)$ and a wrench $W_{m}^{\prime}$ on screw $S_{m}^{\prime}$ representing a pure moment $\left(\lambda_{m}^{\prime}=\infty\right)$ if and only if:

- axes $(\boldsymbol{\Delta})$ and $(\boldsymbol{\Delta})_{f}^{\prime}$ are parallel (i.e., $S_{f}^{\prime}$ is obtained by translating $S$ a certain distance $\left(r_{f}^{\prime}-r\right)$ ), and

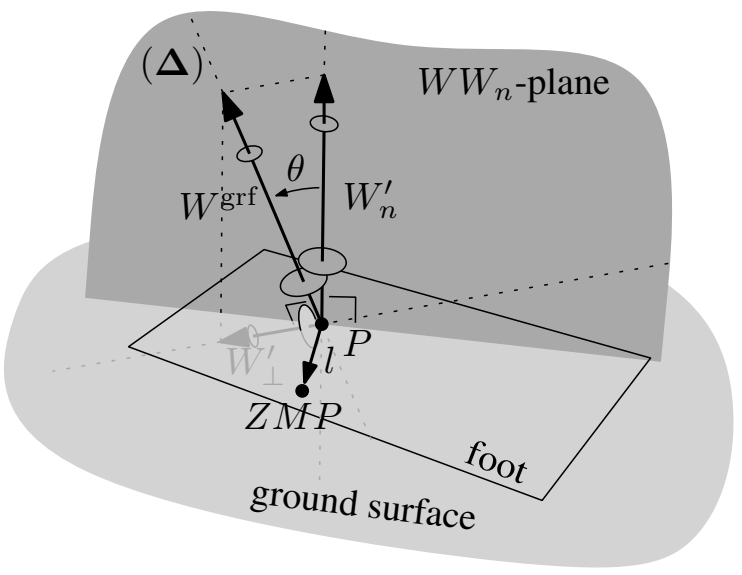

Fig. 6. Geometric construction of the Zero-Moment Point from the ground contact wrench $W^{\text {grf }}$

- the translation vector $\left(r_{f}^{\prime}-r\right)$ is such that

$$
\left(\omega \wedge\left(r_{f}^{\prime}-r\right)+\lambda \omega\right) \| \omega_{m}^{\prime} .
$$

Proof: A valid decomposition can be made if and only if there exist (pseudo-)intensities $v_{f}^{\prime}$ and $v_{m}^{* \prime}$ such that $W=$ $W_{f}^{\prime}+W_{m}^{\prime}$ :

$$
v\left(\begin{array}{c}
r \wedge \omega \\
\omega
\end{array}\right)+v \lambda\left(\begin{array}{c}
\omega \\
0
\end{array}\right)=v_{f}^{\prime}\left(\begin{array}{c}
r_{f}^{\prime} \wedge \omega_{f}^{\prime} \\
\omega_{f}^{\prime}
\end{array}\right)+v_{m}^{* \prime}\left(\begin{array}{c}
\omega_{m}^{\prime} \\
0
\end{array}\right) .
$$

The lower line of (8) implies that $v \omega=v_{f}^{\prime} \omega_{f}^{\prime}$, thus (because $\omega$ and $\omega_{f}^{\prime}$ are unit vectors) $\omega_{f}^{\prime}= \pm \omega$ and $v_{f}^{\prime}= \pm v$. Again, without loss of generality we may assume that $\omega$ and $\omega_{f}^{\prime}$ have the same direction (thus $v=v_{f}^{\prime}$ ). The top line of (8) can now be written as

$$
\left(r-r_{f}^{\prime}\right) \wedge \omega+\lambda \omega=\frac{v_{m}^{* \prime}}{v} \omega_{m}^{\prime} .
$$

Indeed, a suitable $v_{m}^{* \prime}$ can be found if and only if the left hand side of (9) is parallel to $\omega_{m}^{\prime}$.

\section{CONSTRUCTION OF THE ZMP USING THE GROUND REACTION WRENCH}

If the ground reaction wrench $W^{\text {grf }}$ is known, the ZeroMoment Point can quite easily be found geometrically. First we show how this is done; second we will prove that indeed this algorithm gives us the ZMP.

1) Given the ground contact wrench $W^{\text {grf }}$, on screw $S$ (defined by axis $(\boldsymbol{\Delta})$ and pitch $\lambda$ ), find the point $P$, being the intersection between $(\boldsymbol{\Delta})$ and the ground plane.

2) By using theorem 2, decompose $W^{\text {grf }}$ (which has a force component $f^{\text {grf }}$ and a moment component $m^{\text {grf }}=\lambda f^{\text {grf }}$ ) into a wrench $W_{n}^{\prime}$ normal to the ground surface plus a wrench $W_{\perp}^{\prime}$ perpendicular to $W^{\text {grf }}$, intersecting each other in $P$. The force and moment components of the obtained wrenches are denoted $f_{n}^{\prime}, f_{\perp}^{\prime}$ and $m_{n}^{\prime}, m_{\perp}^{\prime}$ respectively. Note that $m_{n}^{\prime}=\lambda f_{n}^{\prime}, m_{\perp}^{\prime}=\lambda f_{\perp}^{\prime}, W_{n}^{\prime}+W_{\perp}^{\prime}=W^{\text {grf }}$ and thus $f_{n}^{\prime}+f_{\perp}^{\prime}=f^{\text {grf }}$ and $m_{n}^{\prime}+m_{\perp}^{\prime}=m^{\text {grf }}$. 
3) Construct a vector $l$ from $P$, perpendicular to the plane spanned by $W^{\text {grf }}$ and $W_{n}$ (this plane is denoted by $W W_{n}$ ), having magnitude $|l|=\lambda \frac{\left|f_{\perp}\right|}{\left|f^{\text {grf }}\right|}$ (the direction of $l$ follows from applying the right-hand rule, going from $W_{n}$ to $W^{\text {grf }}$ ).

4) Now $l$ points to the Zero-Moment Point.

The whole procedure is also sketched in figure 6 .

Proof: The proof shows that the ground reaction wrench is equivalent to a pure force $\bar{f}$ acting on the ZMP plus a moment $\bar{m}_{n}$ that is perpendicular to the ground surface. We introduce a reference frame $\Psi_{0}$ somewhere in space with an arbitrary orientation, a vector $p$ pointing from its origin to point $P$ and a vector $z=p+l$ pointing from its origin to the supposed position of the ZMP. $\bar{f}$ and $\bar{m}_{n}$ can be written as a wrench as $W^{\bar{f}}=\left(\begin{array}{c}z \wedge \bar{f} \\ \bar{f}\end{array}\right)$ and $W^{\bar{m}_{n}}=\left(\begin{array}{c}\bar{m}_{n} \\ 0\end{array}\right)$ respectively.

Assume that indeed $l$ points to the ZMP, then the above defined $\bar{f}$ and $\bar{m}_{n}$ should exist such that

$$
\begin{aligned}
W^{\text {grf }} & =W^{\bar{f}}+W^{\bar{m}_{n}} \\
\left(\begin{array}{c}
p \wedge f^{\text {grf }} \\
f^{\text {grf }}
\end{array}\right)+\lambda\left(\begin{array}{c}
f^{\text {grf }} \\
0
\end{array}\right) & =\left(\begin{array}{c}
z \wedge \bar{f} \\
\bar{f}
\end{array}\right)+\left(\begin{array}{c}
\bar{m}_{n} \\
0
\end{array}\right) .
\end{aligned}
$$

From this, it follows directly that $\bar{f}=f^{\text {grf }}$. Because $z=$ $p+l$, we can write the top row of (10) as $p \wedge f^{\text {grf }}+\lambda f^{\text {grf }}=$ $p \wedge \bar{f}+l \wedge \bar{f}+\bar{m}_{n}$ which can be simplified to $\left(\bar{f}=f^{\text {grf }}\right)$

$$
\lambda f^{\mathrm{grf}}=l \wedge f^{\mathrm{grf}}+\bar{m}_{n} .
$$

It can be shown that $l \wedge f^{\text {grf }}=m_{\perp}^{\prime}$ by proving the following (sufficient) conditions:

1) $m_{\perp}^{\prime} \perp l-l$ is perpendicular to $W^{\text {grf }}$ and $W_{n}^{\prime}$ and, consequently, to any vector in the plane spanned by them. Now $W_{\perp}^{\prime}$ is in this plane, and thus so is $m_{\perp}^{\prime}$ (which has the same direction as $W_{\perp}^{\prime}$ ).

2) $m_{\perp}^{\prime} \perp f^{\text {grf }}-W_{\perp}^{\prime}$ was constructed perpendicularly to $W^{\text {grf }}$. By construction $m_{\perp}^{\prime} \| W_{\perp}^{\prime}$ and $f^{\text {grf }} \| W^{\text {grf }}$. Therefore we have indeed $m_{\perp}^{\prime} \perp f^{\text {grf }}$.

3) $\left|l \wedge f^{\text {grf }}\right|=\left|m_{\perp}^{\prime}\right|$ - because $l \perp f^{\text {grf }}$, we have $\mid l \wedge$ $f^{\text {grf }}|=| l|\cdot| f^{\text {grf }}\left|=\lambda \frac{\left|f_{\perp}^{\prime}\right|}{\left|f^{\text {grf }}\right|} \cdot\right| f^{\text {grf }}|=| \lambda f_{\perp}^{\prime}|=| m_{\perp}^{\prime} \mid$.

4) $l \wedge f^{\text {grf }}=m_{\perp}^{\prime}$ follows the right-hand rule - by inspection of figure 6 .

By using $l \wedge f^{\text {grf }}=m_{\perp}^{\prime}$ and the identities of step 2 of the ZMP construction algorithm, (11) can be rewritten as

$$
\lambda f^{\text {grf }}=m^{\text {grf }}=m_{\perp}^{\prime}+m_{n}^{\prime}=m_{\perp}^{\prime}+\bar{m}_{n}
$$

so we can conclude that indeed there exists an $\bar{f}=f^{\text {grf }}$ and $\bar{m}_{n}=m_{n}^{\prime}$ (which is perpendicular to the ground surface) that satisfy (10), so indeed $l$ points to the Zero-Moment Point.

Remark 1: Both step 1 and 2 of the algorithm are only possible if $(\boldsymbol{\Delta})$ is not parallel to the ground plane. This is logical because if $(\boldsymbol{\Delta})$ were parallel to the ground plane, the ground does not exert a normal force; i.e., the ground does not support the foot and in that case the ZMP does not exist.

Remark 2: If $(\boldsymbol{\Delta})$ is almost vertical, i.e., the friction forces are much smaller than the normal force, we can approximate the magnitude $|l|$ as follows: let $\theta$ be the angle between $f_{n}^{\prime}$ and $f^{\text {grf }}$ (see figure 6); then $\frac{\left|f_{\perp}^{\prime}\right|}{\left|f^{\text {grf }}\right|}=\tan \theta \approx$ $\theta \Rightarrow|l| \approx \lambda \theta$.

Remark 3: As $l$ is perpendicular to $W_{n}^{\prime}$ (and thus to the ground's normal), it it automatically parallel to the ground surface. Therefore, $l$ points to a point belonging to the ground surface; which is in agreement with the commonly-known fact that the ZMP is a point on the ground surface.

Remark 4: We never stated that the ground should be horizontal. In fact, we did not even draw the reference from which a 'world's horizontal plane' could be deduced. This method works for any orientation of the ground plane (and the orientation does not need to be known either). Of course, in order for the ZMP to make sense, the foot must make full contact with the ground surface and therefore the ground surface under the foot should be flat. It is however no problem to have different (possibly discontinuous) parts of flat ground where the robot steps on.

Remark 5: From a decomposition point of view, the algorithm can be interpreted as follows. The ground reaction wrench consists of a force $f^{\text {grf }}$ and a moment $m^{\text {grf }}$ along the same axis $(\Delta)$. In order to find the ZMP, we want to find a decomposition in a purely vertical moment and a pure force. The moment $m^{\text {grf }}$ can be seen as the composition of a 'wanted' vertical component $m_{n}^{\prime}$ and an 'unwanted' remainder $m_{\perp}^{\prime}$. In order to cancel the latter, we can translate $f^{\text {grf }}$ (according to theorem 3) along $l$, which introduces a canceling moment $-m_{\perp}^{\prime}$. The result is a translated version of $f^{\text {grf }}$ and the sum of all moments $m_{n}^{\prime}+m_{\perp}^{\prime}+\left(-m_{\perp}^{\prime}\right)=m_{n}^{\prime}$. The intersection of the translated $f^{\text {grf }}$ and the ground plane is the ZMP.

\section{EXPLICIT EXPRESSION FOR THE ZMP POSITION, GIVEN THE GROUND REACTION WRENCH}

\section{A. Expression for the $Z M P$}

The purpose of this section is to show that, when the ground reaction force/moment is known as a wrench, the expression for the ZMP is simple. Of course, these equations give the same results as any ZMP other equation. Hence the expression presented here is most useful if the ground reaction force/moment is already available as a wrench (otherwise other ZMP equations are usually more direct and thus faster).

Consider an arbitrary walking robot in single support phase and a reference frame $\Psi_{0}$ attached to the ground surface, such that the frame's xy-plane coincides with the ground surface and the frame's z-axis points out of the ground (note that we do not require the ground surface to be horizontal). Let the ground reaction wrench ${ }^{0} W^{\text {grf }}$ be known (see section VI-B), expressed in frame $\Psi_{0}$. By using (10), we can find an explicit expression for the position of the $\mathrm{ZMP}$, as shown below. The ground reaction wrench can be written as (where we use $f=f^{\text {grf }}$ and $m=m^{\text {grf }}$ )

$$
{ }^{0} W^{\text {grf }}=\left(\begin{array}{c}
{ }^{0} m \\
{ }^{0} f
\end{array}\right)=\left(\begin{array}{c}
{ }^{0} z \wedge{ }^{0} \bar{f} \\
0 \bar{f}
\end{array}\right)+\left(\begin{array}{c}
{ }^{0} \bar{m}_{n} \\
0
\end{array}\right)
$$

where ${ }^{0} z$ (the position of the ZMP expressed in coordinates of $\Psi_{0}$ ) is the unknown. By using ${ }^{0} \bar{f}={ }^{0} f,{ }^{0} z \wedge^{0} \bar{f}=-{ }^{0} \bar{f} \wedge^{0} z$ 
and expanding the cross product, we obtain for the top row of (13)

$$
\left(\begin{array}{c}
{ }^{0} m_{x} \\
{ }^{0} m_{y} \\
{ }^{0} m_{z}
\end{array}\right)=\left(\begin{array}{ccc}
0 & { }^{0} f_{z} & -{ }^{0} f_{y} \\
-{ }^{0} f_{z} & 0 & { }^{0} f_{x} \\
{ }^{0} f_{y} & -{ }^{0} f_{x} & 0
\end{array}\right)\left(\begin{array}{c}
{ }^{0} z_{x} \\
{ }^{0} z_{y} \\
{ }^{0} z_{z}
\end{array}\right)+\left(\begin{array}{l}
{ }^{0} \bar{m}_{n x} \\
{ }^{0} \bar{m}_{n y} \\
{ }^{0} \bar{m}_{n z}
\end{array}\right) .
$$

The chosen position and orientation of $\Psi_{0}$ implies that the coordinates of the ZMP, ${ }^{0} z$, and the normal moment, ${ }^{0} \bar{m}_{n}$, satisfy ${ }^{0} z_{z}=0 ;{ }^{0} \bar{m}_{n x}={ }^{0} \bar{m}_{n y}=0$. By substituting this into (14) and rewriting the first two rows, we obtain the explicit equation for the position of the ZMP expressed in coordinates of frame $\Psi_{0}$ :

$$
{ }^{0} z=\left(\begin{array}{c}
{ }^{0} z_{x} \\
{ }^{0} z_{y} \\
{ }^{0} z_{z}
\end{array}\right)=\left(\begin{array}{c}
-{ }^{0} m_{y} /{ }^{0} f_{z} \\
{ }^{0} m_{x} /{ }^{0} f_{z} \\
0
\end{array}\right)
$$

\section{B. Obtaining the ground reaction wrench}

In a robot, the ground reaction wrench should be measured in some way. An easy way to do this is by utilizing a $6 \mathrm{D}$ force sensor. By placing the reference frame $\Psi_{0}$ in the origin of the sensor, oriented such that the xy-plane is aligned to the sole of the foot, the forces and moments $f_{x}, f_{y}, f_{z}, m_{x}, m_{y}, m_{z}$ are directly the elements of ${ }^{0} W^{\text {grf }}$. The fact that the reference frame may move relatively to the fixed world does not invalidate the results.

In simulation, the ground reaction wrench is usually already available in some form because it is needed anyway for simulating the foot-ground interaction.

\section{CONCLUSIONS}

In this article we have shown that the concept of screws and wrenches gives us tools to geometrically establish the relation between the ground reaction wrench and the ZeroMoment Point. In order to arrive at this, we showed how a wrench can be decomposed into separate components. The proposed method gives a general, completely coordinatefree way to find the ZMP. The power of coordinate-free analysis is broadly and recognized to prevent any kind of implicit (and possibly wrong) assumptions in the analysis. The method contributes to a better insight in the problem and gives support for graphical animation of locomotion.

\section{APPENDIX}

Proof: [A more mathematical proof of theorem 2] The screws $S, S_{1}^{\prime}$ and $S_{2}^{\prime}$ can be represented by axis locations $r$, $r_{1}^{\prime}$ and $r_{2}^{\prime}$, axis directions $\omega, \omega_{1}^{\prime}$ and $\omega_{2}^{\prime}$ and common pitch $\lambda=\lambda_{1}^{\prime}=\lambda_{2}^{\prime}$.

First condition - Without loss of generality we can assume that the location vectors point to the intersection point and thus $r=r_{1}^{\prime}=r_{2}^{\prime}$. The condition that $\omega_{1}^{\prime}$ and $\omega_{2}^{\prime}$ span a plane which contains $\omega$ implies that there exist an $\alpha$ and $\beta$ such that $\omega=\alpha \omega_{1}^{\prime}+\beta \omega_{2}^{\prime}$. Now by choosing intensities $v_{1}^{\prime}=v \alpha$ and $v_{2}^{\prime}=v \beta$, we can write

$$
\begin{gathered}
W_{1}^{\prime}+W_{2}^{\prime}= \\
v_{1}^{\prime}\left(\left(\begin{array}{c}
r_{1}^{\prime} \wedge \omega_{1}^{\prime} \\
\omega_{1}^{\prime}
\end{array}\right)+\lambda_{1}^{\prime}\left(\begin{array}{c}
\omega_{1}^{\prime} \\
0
\end{array}\right)\right)+v_{2}^{\prime}\left(\left(\begin{array}{c}
r_{2}^{\prime} \wedge \omega_{2}^{\prime} \\
\omega_{2}^{\prime}
\end{array}\right)+\lambda_{2}^{\prime}\left(\begin{array}{c}
\omega_{2}^{\prime} \\
0
\end{array}\right)\right)= \\
\left(\begin{array}{c}
r \wedge\left(v_{1}^{\prime} \omega_{1}^{\prime}+v_{2}^{\prime} \omega_{2}^{\prime}\right) \\
v_{1}^{\prime} \omega_{1}^{\prime}+v_{2}^{\prime} \omega_{2}^{\prime}
\end{array}\right)+\lambda\left(\begin{array}{c}
v_{1}^{\prime} \omega_{1}^{\prime}+v_{2}^{\prime} \omega_{2}^{\prime} \\
0
\end{array}\right)= \\
v\left(\left(\begin{array}{c}
r \wedge \omega \\
\omega
\end{array}\right)+\lambda\left(\begin{array}{c}
\omega \\
0
\end{array}\right)\right)=W
\end{gathered}
$$

which shows that indeed there exists a decomposition.

Second condition - All axes are parallel, thus $\omega=\omega_{1}^{\prime}=$ $\omega_{2}^{\prime}$. Without loss of generality we can assume that $r, r_{1}^{\prime}$ and $r_{2}^{\prime}$ lie on one line, thus there exists an $\alpha$ such that $r=$ $\alpha r_{1}^{\prime}+(1-\alpha) r_{2}^{\prime}$. Now by choosing $v_{1}^{\prime}=v \alpha, v_{2}^{\prime}=v(1-\alpha)$, we can write

$$
\begin{gathered}
W_{1}^{\prime}+W_{2}^{\prime}= \\
v_{1}^{\prime}\left(\left(\begin{array}{c}
r_{1}^{\prime} \wedge \omega_{1}^{\prime} \\
\omega_{1}^{\prime}
\end{array}\right)+\lambda_{1}^{\prime}\left(\begin{array}{c}
\omega_{1}^{\prime} \\
0
\end{array}\right)\right)+v_{2}^{\prime}\left(\left(\begin{array}{c}
r_{2}^{\prime} \wedge \omega_{2}^{\prime} \\
\omega_{2}^{\prime}
\end{array}\right)+\lambda_{2}^{\prime}\left(\begin{array}{c}
\omega_{2}^{\prime} \\
0
\end{array}\right)\right)= \\
\left(\begin{array}{c}
\left(v_{1}^{\prime} r_{1}^{\prime}+v_{2}^{\prime} r_{2}^{\prime}\right) \wedge \omega \\
\left(v_{1}^{\prime}+v_{2}^{\prime}\right) \omega
\end{array}\right)+\left(v_{1}^{\prime}+v_{2}^{\prime}\right) \lambda\left(\begin{array}{c}
\omega \\
0
\end{array}\right)= \\
v\left(\left(\begin{array}{c}
r \wedge \omega \\
\omega
\end{array}\right)+\lambda\left(\begin{array}{c}
\omega \\
0
\end{array}\right)\right)=W
\end{gathered}
$$

which shows that indeed there exists a decomposition.

\section{REFERENCES}

[1] M. Vukobratović and B. Borovac, "Zero-Moment Point - thirty five years of its life," International journal of humanoid robotics, vol. 1, no. 1, pp. 157-173, 2004.

[2] R. Ball, A treatise on the theory of screws. Cambridge University Press, Cambridge, 1900.

[3] S. Takao, Y. Yokokohji, and T. Yoshikawa, "FSW (feasible solution of wrench) for multi-legged robots," in Robotics and Automation. (ICRA '03). Proc. IEEE Int. Conf. on, vol. 3, Sept. 2003, pp. 3815-3820.

[4] J. Park, Y. Youm, and W. Chung, "Control of ground interaction at the zero-moment point for dynamic control of humanoid robots," in Robotics and Automation, 2005. ICRA 2005. Proceedings of the 2005 IEEE International Conference on, April 2005, pp. 1724-1729.

[5] P. Sardain and G. Bessonnet, "Forces acting on a biped robot. center of pressure-zero moment point," Systems, Man and Cybernetics, Part A: Systems and Humans, IEEE Transactions on, vol. 34, no. 5, pp. $630-637$, Sept. 2004.

[6] M. Vukobratović and D. Juričić, "Contribution to the synthesis of biped gait," Proc. IFAC Symp. Technical and Biological Problem and Control, 1968.

[7] — "Contribution to the synthesis of biped gait," Biomedical Engineering, IEEE Transactions on, vol. 16, no. 1, pp. 1-6, Jan. 1969.

[8] A. Dasgupta and Y. Nakamura, "Making feasible walking motion of humanoid robots from human motion capture data," in ICRA, 1999, pp. 1044-1049.

[9] K. Harada, S. Kajita, K. Kaneko, and H. Hirukawa, "ZMP analysis for arm/leg coordination," in Intelligent Robots and Systems, 2003. (IROS 2003). Proc. IEEE/RSJ Int. Conf. on, vol. 1, 2003, pp. 75-81.

[10] M. Vukobratović, B. Borovac, and D. Surdilovic, "Zero-moment point - proper interpretation and new applications," Int. Conf. on Humanoid Robots, 2001.

[11] A. Goswami, "Postural stability of biped robots and the Foot-Rotation Indicator (FRI) point," Int. J. Robotic Research, vol. 18, no. 6, pp. 523-533, 1999.

[12] J. E. Pratt and R. Tedrake, "Velocity-based stability margins for fast bipedal walking," pp. 299-324, 2006.

[13] R. M. Murray, Z. Li, and S. S. Sastry, A Mathematical Introduction to Robotic Manipulation. CRC, Mar. 1994.

[14] R. Featherstone, Rigid Body Dynamics Algorithms. Secaucus, NJ, USA: Springer-Verlag New York, Inc., 2007. 